\title{
LIPOSSARCOMA EM CATURRITA (Myiopsitta monachus)
}

\author{
Anelise Bonilla Trindade, ${ }^{1}$ Maurício Veloso Brun, ${ }^{2}$ Fernando Bilibio Riviera, ${ }^{3}$ \\ Paula Cristina Basso, ${ }^{4}$ Stella Faria Valle ${ }^{5}$ e Emerson Antonio Contesini ${ }^{6}$
}

\footnotetext{
1. Doutoranda, Programa de Pós-Graduação em Ciências Veterinárias na Universidade Federal do Rio Grande do Sul (UFRGS)

2. Médico veterinário, doutor, professor na Universidade Federal de Santa Maria (UFSM), professor do Mestrado em Medicina de Pequenos Animais da Universidade de Franca (UNIFRAN)

3. Médico veterinário, autônomo

4. Doutoranda, Programa de Pós-Graduação em Medicina Veterinária da Universidade Federal de Santa Maria (UFSM)

5. Mestre, professora na Universidade de Passo Fundo (UPF)

6. Médico veterinário, doutor, professor na Universidade Federal do Rio Grande do Sul (UFRGS).
}

RESUMO

Os lipossarcomas são tumores malignos de lipócitos e lipoblastos raramente relatados em espécies aviárias. Acometem geralmente pássaros adultos ou velhos e em regiões onde existe acúmulo de tecido adiposo. O presente trabalho relata um caso raro de lipossarcoma em uma caturrita (Myiopsitta monachus) com cinco anos de idade que apresentava nódulo de crescimento progressivo na extremidade distal da asa esquerda, com evolução de seis meses. Apesar da dificuldade de deambulação, a ave apresentava disposição e normorexia. Procedeu-se à extirpação cirúrgica da massa tumoral, e o exame histopatológico permitiu o diagnóstico de lipossarcoma bem diferenciado. Não foram observadas recidivas ou metástases durante o período de 24 meses após o procedimento, o que confirma a adequação da conduta cirúrgica realizada.

PALAVRAS-CHAVES: Cirurgia, lipossarcoma, neoplasia, pássaros de estimação.

\section{ABSTRACT}

\section{LIPOSARCOMA IN A QUAKER PARROT (Myiopsitta monachus) - CASE REPORT}

Liposarcomas are malignant tumors that involve lipocytes and lipoblasts. This neoplasm is not frequently reported in avian species and generally occurs in adult or old birds and it is always seen in areas of adipose accumulation. This report presents a rare case of liposarcoma in a five-year-old quaker parrot (Myiopsitta monachus). The patient had a 6-month progressive growing nodule

KEYWORDS: Liposarcoma, pet birds, surgery, tumors.

\section{INTRODUÇÃO}

Os lipossarcomas são tumores malignos de lipócitos e lipoblastos raramente relatados em pássaros de estimação (REAVILL, 2004). Ocorrem geralmente em animais adultos ou velhos (MOULTON, 1990) e são at the left wing extremity and ataxia; however, normal appetite and disposition were noticed. After the neoplasm extirpation, the pathological study pointed out a well-differentiated liposarcoma. Local disease or metastasis signals were not observed within 24 months after the surgery. The surgical procedure was effective to control the liposarcoma in this bird.

vistos quase sempre em locais onde existe acúmulo de tecido adiposo (ABELOFF et al., 2000; ROSENBERG, 2000). Apesar de existir associação à presença de corpo estranho, sua etiologia não é conhecida e acredita-se que não resulta da transformação maligna de lipomas preexistentes (McCARTHY et al., 1996). 
A localização anatômica dos lipossarcomas, descrita em pássaros, inclui a região que circunda o carpo, membros pélvicos, dígitos, pescoço, tecido subcutâneo esternal, glândula uropigeana e cavidade abdominal, porém metástases podem ser encontradas no fígado (DOSTER et al., 1987; REAVILL, 2001). Tais tumores apresentam-se normalmente como massas subcutâneas amarelo-pálido, firmes e mais infiltrativos que os lipomas (PETRAK \& GILMORE, 1982; REECE, 1992; REAVILL, 2001).

Assim como outros tumores mesenquimais, os lipossarcomas tendem a ser localmente invasivos, porém possuem capacidade metastática baixa (McCARTHY et al., 1996). Segundo KRANSDORF et al. (1993), LEITCH (1993), PRIOLLI et al. (2003), esses neoplamas são classificados conforme o grau de diferenciação celular em: grau I- bem diferenciado; grau II- mixoide; grau III- pleomórfico; grau IV- com células arredondadas; Grau V- tipo fibroblástico.

Pelo fato de o lipossarcoma ser um neoplasma raro, principalmente em aves, o presente trabalho visa relatar um caso deste envolvendo uma caturrita (Myiopsitta monachus), em que se descrevem os sinais clínicos apresentados por ela, diagnóstico e tratamento realizados. Ressalta-se que os autores não encontraram relatos na literatura que citem a ocorrência deste neoplasma em caturritas.

\section{MATERIAL E MÉTODOS}

Uma caturrita adulta, com cinco anos de idade e $142 \mathrm{~g}$ de massa corporal, foi atendida no Hospital Veterinário da Universidade de Passo Fundo (UPF), com histórico de presença de nódulo na extremidade da asa esquerda com crescimento progressivo havia seis meses, o qual passou a dificultar sua deambulação. Apesar disso, o animal apresentava normorexia e boa disposição.

A ave já havia sido tratada anteriormente em outro estabelecimento veterinário, no qual foi sugerido o diagnóstico de lipoma, sem exames cito ou histopatológicos prévios. Naquela ocasião, foi iniciado o tratamento com dexametasona ${ }^{1}(1 \mathrm{mg} / \mathrm{kg})$ por via oral, a cada oito horas, durante seis dias, com redução gradativa da dose.

Ao exame clínico, constatou-se massa na região falangeana esquerda (Figura 1), com aproximadamente quatro centímetros de diâmetro, móvel, indolor, pediculada, e de consistência fibroelástica. Apresentava algumas penas em seu interior e com grande ulceração superficial na porção medial. Foram realizados exames radiográficos do membro acometido, os quais não revelaram envolvimento ósseo.

Foi indicado o tratamento com enrofloxacina ${ }^{2}$ (15 mg/kg, BID,VO) e com cetoprofeno ${ }^{3}(2 \mathrm{mg} / \mathrm{kg}$, VO SID), ambos por cinco dias consecutivos. Houve reavaliação da paciente, sendo encaminhada para a exérese da massa tumoral.

Como medicação pré-anestésica, utilizou-se butorfanol $^{4}$ (1mg, IM), sendo a indução e manutenção da anestesia com isoflurano ${ }^{5}$ vaporizado em $\mathrm{O}_{2}$ a $100 \%$, em circuito semiaberto, a $0,6 \mathrm{~L} / \mathrm{min}$. Para a ressecção tumoral, retiraram-se manualmente algumas penas que se encontravam na base do tumor. A antissepsia foi realizada com clorexidine ${ }^{6}$ aquoso a $0,05 \%$. Posteriormente, efetuou-se incisão elíptica na base do tumor, constatando-se sangramento abundante para a espécie e tamanho do animal. A hemostasia foi obtida por compressão manual, realizando-se, posteriormente, sutura contínua simples da pele com fio poliglactina 910 3-0. $\mathrm{O}$ animal recebeu monitoração e aquecimento até sua completa recuperação anestésica. O nódulo excisado foi encaminhado para análise histopatológica, a qual revelou lipossarcoma bem diferenciado.

No pós-operatório, administrou-se cetoprofeno $(2 \mathrm{mg} / \mathrm{kg}$, IM, SID) por três dias consecutivos e efetuouse limpeza da ferida cirúrgica com solução de $\mathrm{NaCl}$ a $0,9 \%$ três vezes ao dia, durante oito dias. A ave recebeu alta hospitalar três dias após o procedimento cirúrgico.

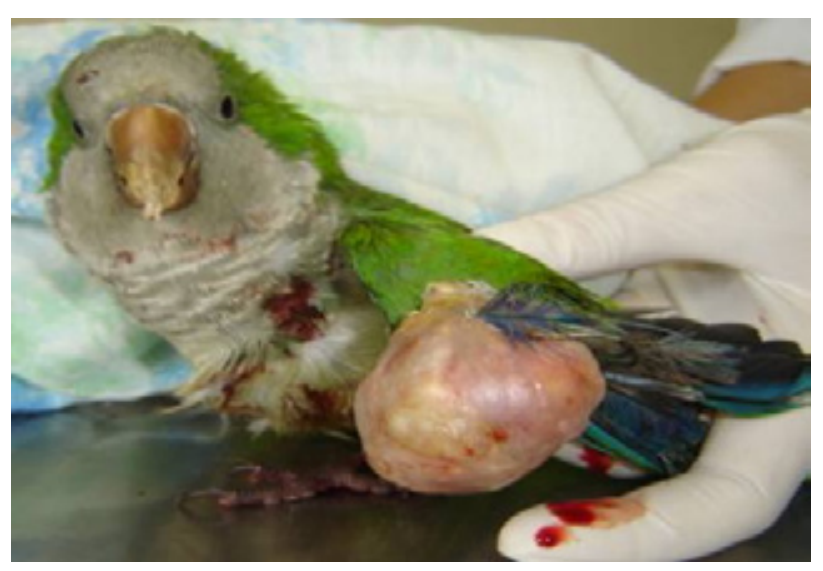

FIGURA 1. Caturrita com lipossarcoma bem diferenciado. Observar massa na região falangeana esquerda. 


\section{RESULTADOS E DISCUSSÃO}

Os sarcomas de partes moles são tumores incomuns em humanos e animais (GRAHAM et al., 2003; PRIOLLI et al., 2003), sendo o lipossarcoma um neoplasma maligno de adipócitos que acomete raramente os animais domésticos (GOLDSHIMITH \& HENDRICK, 2002; BAEZ et al., 2004), ao contrário do lipoma, que é uma das neoplasias mesenquimais benignas mais frequentes em pequenos pássaros (GOLDSHIMITH \& HENDRICK, 2002; REAVIL, 2004). LOPES et al. (1996), NASCIMENTO \& OLIVEIRA (1999) e ABELOFF et al. (2000) relatam que, em humanos, as lesões são geralmente únicas e dificilmente atingem grandes proporções. Porém, tumores de grandes proporções são vistos em regiões corpóreas superficiais com menor frequência (NUCCI \& FLETCHER, 1998). No presente caso, a neoplasia apresentava dimensões consideráveis a ponto de impedir a deambulação normal da ave, sendo tal fato justificado pelo tempo relativamente longo de evolução da doença.

O diagnóstico clínico geralmente é difícil, e na maioria das vezes é comum o diagnóstico errôneo de lipoma (REAVIL, 2004), fato este também observado na ave analisada, em que primeiramente foi indicado dexametasona. O uso inicial deste fármaco, com redução gradual da dose, não foi justificado, visto que o tratamento usual do lipoma consiste na sua remoção cirúrgica conforme descrito por LUZ (2006). Este mesmo autor ainda cita que técnicas cirúrgicas adequadas permitem que mesmo lipomas grandes possam ser removidos por meio de pequenas incisões.

A referida ave doméstica foi encaminhada para a exérese da massa tumoral, sem prévio exame citopatológico e/ou histopatológico, concordando com REAVILL (2004), o qual afirma que a biopsia aspirativa pode não diferenciar lipossarcoma de lipoma. Cabe ressaltar que, em virtude de a neoplasia apresentar áreas de aspecto histológico variável e de difícil interpretação, a quantidade de material a ser examinado deverá ser substancial, preconizando-se, então, a utilização de agulhas de grosso calibre em aspirações ou a não realização deste procedimento (GILL, 1999; PRIOLLI, 2003). Optou-se, portanto, pela não realização da biopsia aspirativa e/ou inci- sional, evitando-se, assim, estresse desnecessário ao animal.

GRAHAM et al. (2003) realizaram biopsia aspirativa com agulha fina em um papagaio-cinza (Psittacus erithacus) e os resultados revelados foram inconclusivos, em virtude do rompimento das células durante a colheita do material. Estes mesmos autores ainda citam que não é recomendada a realização da biopsia incisional em casos de suspeita de lipossarcoma, pelo alto potencial de disseminar células tumorais no animal.

O nódulo excisado foi encaminhado para análise histopatológica, sendo que a avaliação macroscópica revelou dimensões de 3,5 x 3,0 x 3,5 cm e coloração amarelo-esbranquiçada. $\mathrm{O}$ exame microscópico demonstrou proliferação de adipócitos neoplásicos, dispostos em mantos, entremeados por escasso estroma conjuntivo. Em algumas áreas, as células tumorais invadiam focalmente a epiderme. Os adipócitos neoplásicos tinham tamanhos variados, com macro e/ ou microvacúolos intracitoplasmáticos (Figura 2). Os núcleos eram periféricos e muitas vezes pleomórficos, variando entre arredondados, ovais ou alongados, com um a três nucléolos (Figura 3). Havia extensas áreas de necrose, infiltradas por heterófilos e contendo aglomerados de bactérias basofílicas intralesionais, firmando o diagnóstico morfológico de lipossarcoma bem diferenciado.

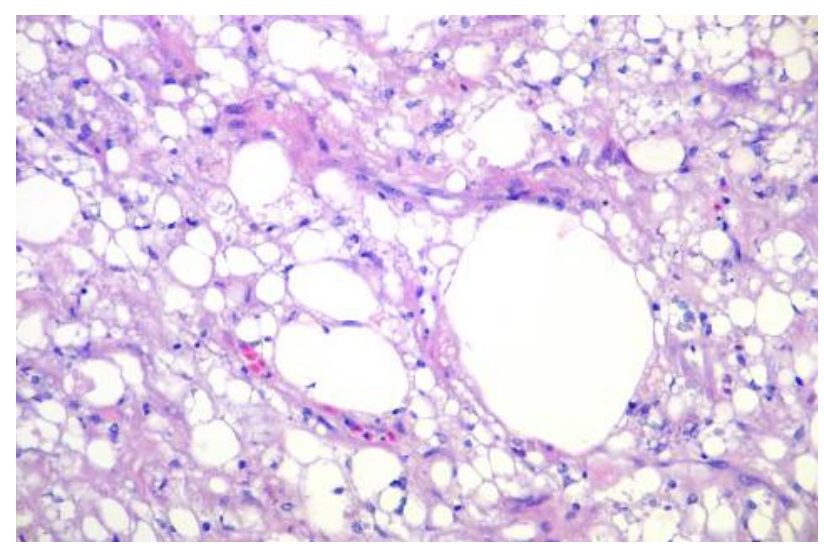

FIGURA 2. Exame histológico da massa extirpada da caturrita diagnosticada como lipossarcoma bem diferenciado. Observam-se adipócitos neoplásicos de tamanhos variados, contendo vacúolos citoplasmáticos. O estroma tumoral é escasso. Hematoxilinaeosina. Obj. 20x. 


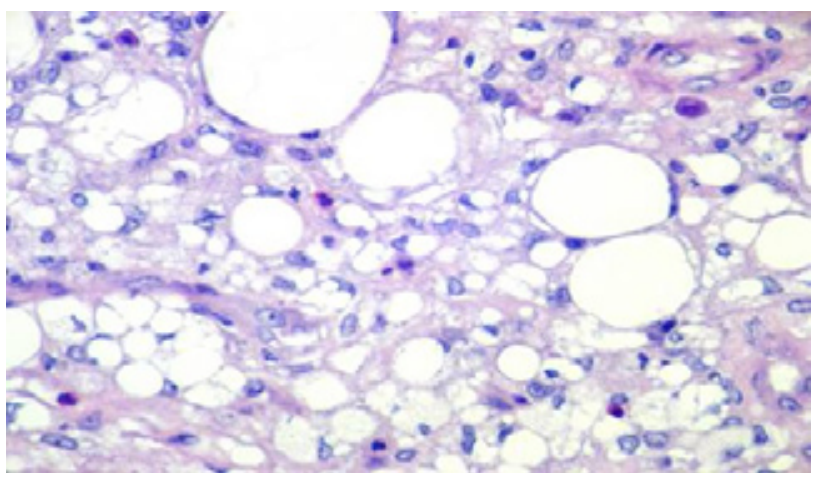

FIGURA 3. Exame histológico da massa extirpada da caturrita, diagnosticada como lipossarcoma bem diferenciado. Caturrita, lipossarcoma bem diferenciado. Observam-se adipócitos neoplásicos com núcleos excêntricos e pleomórficos, contendo um a três núcleolos evidentes. Hematoxilina-eosina. Obj. 40x.

As características da massa tumoral da presente ave relatada estão de acordo com LOPES et al. (1996), os quais citaram que em humanos a manifestação clínica na maior parte dos casos é a presença de massa indolor que progressivamente aumenta de tamanho, abaulando a pele subjacente, tornando-a lisa e brilhante. Sabe-se que na medida em que o tumor progride, em virtude de fenômenos isquêmicos ou pela infiltração neoplásica, a pele adquire tonalidade escurecida, evoluindo com frequência para necrose e ulceração (LOPES et al., 1996), condição observada no paciente em questão, a qual foi agravada pelo uso inicial de dexametasona. A utilização do glicocorticoide favoreceu o aumento na ulceração do tumor na ave, uma vez que estes fármacos afetam a síntese e maturação do colágeno, alteram a força de tensão das feridas, inibem a função do fibroblasto e deprimem a ação antibacteriana e fagocitária de algumas células de defesa, resultando na alteração do padrão e retardo da cicatrização das feridas (KLOTH $\&$ McCULLOCH, 1998). Isso é corroborado com as observações do presente caso, em que havia a presença de bactérias intralesionais na massa tumoral do paciente relatado, sugerindo a existência de infecção secundária, provavelmente em decorrência de lesão traumática, agravada pelo uso concomitante de dexametasona.

A radiografia realizada na ave desse relato permitiu descartar um possível envolvimento ósseo. Conduta semelhante foi realizada por OLIVEIRA et al. (2006), ao verificarem um nódulo em um pa- pagaio-verdadeiro (Amazona aestiva), em que, posteriormente, a análise histopatológica confirmou lipoma nessa espécie. Recentemente, OCARINO et al. (2005) relataram um caso de lipossarcoma ósseo em cão, com intensa reação periosteal. Esses autores referem como possível explicação para essa reação o fato de em alguns processos neoplásicos as células tumorais liberarem fatores que promovem o crescimento do tecido neoplásico e até mesmo de tecido ósseo normal adjacente. Porém, essas alterações ainda não foram relatadas em aves com lipossarcoma (GRAHAM et al., 2003).

O diagnóstico definitivo é baseado no padrão histológico, que é muito variável, e sua classificação depende do comportamento biológico da neoplasia, considerando-se os subtipos previamente citados. O subtipo mixoide, quando diagnosticado precocemente, apresenta prognóstico favorável, o que se deve ao seu baixo potencial para metástases e à sua consistência que aparentemente facilita a remoção cirúrgica completa (LEWIS, 1991), o que está de acordo com o laudo histopatológico e evolução clínica pósoperatória do paciente relatado.

Relatos em humanos citam que a velocidade de crescimento deste tipo de neoplasia está diretamente relacionada ao seu grau de diferenciação celular. Tumores de baixo grau apresentam crescimento lento, infiltrando os tecidos e órgãos vizinhos (GILL, 1999). De acordo com as características da neoplasia, bem como a partir do laudo histopatológico e evolução do paciente, acredita-se tratar-se de um tumor de baixo grau de malignidade. Tumores de estágio I de malignidade são tratados à semelhança do que foi realizado neste estudo, com ressecção local, respeitando-se margens cirúrgicas adequadas (PRIOLLI et al., 2003).

Como há grande semelhança clínica e macroscópica entre lipomas e lipossarcomas, médicos veterinários devem estar atentos quanto à melhor forma de diagnosticar essas neoplasias, bem como a conduta adequada e orientação, quanto ao prognóstico do animal, principalmente em pássaros de estimação.

\section{CONCLUSÃO}

A remoção cirúrgica do lipossarcoma na caturrita relatada foi efetiva no tratamento dessa patologia, visto que a evolução pós-operatória da 
presente ave, sem evidências de recidivas, um ano após a operação. O procedimento cirúrgico respalda a adequabilidade da conduta realizada.

\section{AGRADECIMENTOS}

À professora Dra Gláucia Kommers, pela orientação e ajuda na interpretação das análises histopatológicas.

\section{FONTES DE AQUISIÇÃO}

${ }^{1}$ Dexametasona, Laboratório Eurofarma Ltda., São Paulo, SP; ${ }^{2}$ Baytril ${ }^{\circledR}$, Laboratório Bayer, São Paulo, SP; ${ }^{3}$ Ketofen ${ }^{\circledR}$, Laboratório Merial, Campinas, SP. ${ }^{4}$ Turbogesic $\AA$, Laboratório Fort Dodge, Campinas, SP; ${ }^{5}$ Forane ${ }^{\circledR}$, Laboratório Abbott, São Paulo, SP; ${ }^{6}$ Clorexidine $\AA$, Laboratório Rioquímica Ltda., São Paulo, SP.

\section{REFERÊNCIAS}

ABELOFF, M. D.; ARMitAge, J. O.; LiCHTER, A. S.; NIEDERHUBER, J. E. Clinical oncology. 2. ed. New York: Churchill Livingstone, 2000. p. 1963.

BAEZ, J. L.; HENDRICK, M. J.; SHOFER, F. S.; GOLDKAMP, C.; SORENMO, K. U. Liposarcomas in dogs: 56 cases. (19892000). Journal American Veterinary Medical Association, v. 224, n. 6, p. 887-891, mar. 2004.

DOSTER, A.; JOHNSON, J.; DUHAMEL, G. E.; BARGAR, T. W.; NASON, G. Liposarcoma in a Canada goose (Branta Canadensis). Avian Diseases, v. 31, n. 4, p. 918-920, Oct-Dec., 1987.

GILL, P. G. Sarcomas de partes moles. In: HOSSFELD, D. K.; SHERMAN, C. D.; LOVE, R. D.; BOSCH, F. X. Manual de oncologia clínica. 6. ed. São Paulo: Fundação Oncocentro de São Paulo, 1999. p. 469-476.

GOLDSHIMITH, M. H.; HENDRICK, M. J. Tumours of the skin and soft tissues. In: MEUTEN, D. Tumors in domestic animals. Iowa State: University of California Press, 2002. 788 p.

GRAHAM, J. E.; WERNER, J. A.; LOWENSTINE, L. J.; WALLACK, S. T.; TELL, L. A. Periorbital liposarcoma in an African Grey Parrot (Psittacus erithacus). Journal of Avian Medicine and Surgery, v. 17, n. 3, p. 147-153, 2003.

KLOTH, L. C.; McCULLOCH, J. M. The inflammatory response to wounding. In: McCULLOCH, J.C, KLOTH, L.C, FEEDAR, J.A. Wound healing alternatives in management. 2. ed. Philadelphia: CPR F.A. Company, 1998. p. 3-15.

KRANSDORF, M.; MEIS, J. M.; JELINEK, J. S. Dedifferentiated liposarcoma of the extremities: imaging findings in four patients. American Journal Roentgenology, v. 1, n. 161, p. 127-131, 1993.

LEITCH, A. Sarcomas and lymphomas: an overview. Selected Readings in General Surgery, v. 20, p. 1-5, 1993.

LEWIS, D. D. Extradural spinal liposarcoma in a dog. Journal American Veterinary Medical Association, v. 199, n. 11, p. 1606-1607, dez. 1991.

LOPES, A.; ROSSI, B. M.; NAKAGAWA, W. T. Sarcoma de partes moles no adulto. In: KOWALSKI, L.P.; SABBAGA, J.; FOGAROLI, R.C. Manual de condutas diagnósticas e terapêuticas em oncologia. São Paulo: Âmbitos, 1996. p. 489-500.

LUZ, F. Doenças da pele. Disponível em: $<$ http://www.dermatologia.net/neo/base/doecas/lipoma.htm> Acesso em 25 jul. 2006

McCARTHY, P. E.; HEDLUND, C. S.; VEAZY, R. S.; PRESCOTTMETHEWS, J.; CHO, D. Y. Liposarcoma associated with a glass foreign body in a dog. Journal American Veterinary Medical Association, v. 209, n. 3, p. 612-614, 1996.

MOULTON, J.E.; HARVEY, J. W. Tumors of the lymphoid and hemopoietic tissues. In: MOULTON, J.E. Tumors in domestic animals. Berkeley: University of California, 1990. p. 23-82.

NASCIMENTO, A.; OLIVEIRA, A. M. Sarcoma de partes moles. In: LOPES, A. Patologia geral. Rio de Janeiro: Medsi, 1999. p. 41-68.

NUCCI, M. R.; FLETCHER, C. D. Liposarcoma (atypical lipomatous tumors) of the vulva: a clinicopathologic study of six cases. International Journal of Gynecological Pathology, v. 17, n. 1, p. 17-23, jan. 1998.

OCARINO, N.M.; SILVA, A. E.; MELO, E. G.; FRANÇA, S. A.; PAIXAO, T. A.; SERAKIDES, R. Lipossarcoma ósseo em cão: relato de caso. Clínica Veterinária, v. n. 59, p. 36-40, nov-dez. 2005.

OLIVEIRA, F. S.; MARTINS, L. L.; DELFINI, A., FARIA Jr., D. Exérese de lipoma em papagaio-verdadeiro (Amazona aestiva). Acta Scientiae Veterinariae, v. 34, n. 2, p. 201-203, 2006.

PETRAK, M.; GILMORE, C. Neoplasms In: PETRAK, M. Diseases of cage and aviary birds. Philadelphia, P.A: Lea \& Febiger, 1982. p. 606-637.

PRIOLLI, D. G.; MARTINEZ, C. A. R.; CAMPIONE, F.; 
ARAÚJO, J. G. L.; WAISBERG, J.; MAGRINI, E. Lipossarcoma gigante da vulva. Revista Brasileira de Ginecologia e Obstetrícia, v. 25, n. 2 , p. 131-135, 2003.

REAVILL, D. R. Tumors of pet birds. Veterinary Clinics of North America: Exotic Animal Pratice, v. 7, n. 3, p. 537-560, 2004.
REECE, R. L. Observations on a naturally occurring neoplasms in birds in the state of Victoria, Australia. Avian Pathology, v. 21, p. 3-321, 1992.

ROSENBERG, A. Ossos, articulações e tumores de partes moles. In: COTRAN, R.S.; KUMAR, V.; COLLINS, T. Robbins: Patologia estrutural e funcional. Rio de Janeiro: Guanabara Koogan, 2000. p. 1087-1134.

Submetido em: 2 maio 2008. Aceito em: 8 out. 2008. 